\title{
Change detection and occlusion modes in road-traffic scenarios
}

\author{
Boris M. Velichkovsky ${ }^{\mathrm{a}, *}$, Sascha M. Dornhoefer ${ }^{\mathrm{a}}$, Mathias Kopf ${ }^{\mathrm{b}}$, \\ Jens Helmert ${ }^{\text {a }}$, Markus Joos ${ }^{\text {a }}$ \\ a Applied Cognitive Research Unit/Psychology III, Dresden University of Technology, 01062 Dresden, Germany \\ b $B M W A G, 80788$ Munich, Germany
}

Received 15 February 2002; received in revised form 19 March 2002; accepted 20 March 2002

\begin{abstract}
Change blindness phenomena are widely known in cognitive science, but their relation to driving is not quite clear. We report a study where subjects viewed colour video stills of natural traffic while eye movements were recorded. A change could occur randomly in three different occlusion modes-blinks, blanks and saccades - or during a fixation (as control condition). These changes could be either relevant or irrelevant with respect to the traffic safety. We used deletions as well as insertions of objects. All occlusion modes were equivalent concerning detection rate and reaction time, deviating from the control condition only. The detection of relevant changes was both more likely and faster than that of irrelevant ones, particularly for relevant insertions, which approached the base line level. Even in this case, it took about $180 \mathrm{~ms}$ longer to react to changes when they occurred during a saccade, blink or blank. In a second study, relevant insertions and the blank occlusion were used in a driving simulator environment. We found a surprising effect in the dynamic setting: an advantage in change detection rate and time with blanks compared to the control condition. Change detection was also good during blinks, but not in saccades. Possible explanation of these effects and their practical implications are discussed.
\end{abstract}

(C) 2002 Elsevier Science Ltd. All rights reserved.

Keywords: Attention; Change blindness; Driving; Eye movements; Blinks; Hazard perception; Masking

\footnotetext{
${ }^{*}$ Corresponding author. Tel.: +49-351-463-34221; fax: +49-351-463-37741.
}

E-mail address: velich@applied-cognition.org (B.M. Velichkovsky). 


\section{Introduction}

\subsection{Change blindness}

A number of recent studies have shown that surprisingly large changes to details of a visual scene often go unnoticed - even if the observers are actively searching for the changes or directly looking at places where they occur (see e.g. Simons \& Levin, 1997). This "change blindness" is typically related to natural global disruptions of the retinal image, such as saccades (Grimes, 1996) or eyeblinks (O'Regan, Deubel, Clark, \& Rensink, 2000). For example, observers could study everyday scenes presented for a later recognition (Grimes, 1996). While they freely explored the scenes, some details were changed in a gaze-contingent manner (e.g. two people exchanging heads or a prominent building in a skyline becoming $25 \%$ larger). A considerable number of changes $(67 \%)$, that could cover one-fourth of the picture, sometimes in the very center of visual field, were missed. The same changes were easily detected when they occurred during a fixation.

Change blindness is not specifically related to saccades or blinks, however: it can also occur during artificial global disruptions, such as a blank screen, where a saccadic suppression or a blink is simulated by a brief blank screen that is inserted between the original and the modified picture using either a forced choice (e.g. Simons, 1996) or a “flicker” paradigm (Rensink, O'Regan, \& Clark, 1997). In forced choice tasks, the original and the modified picture are each presented for one view before the observers have to guess whether a change had occurred. In the flicker paradigm (it has a less natural character and may interfere with different aspects of perceptual processing — see, e.g. Macknik, Fisher, \& Bridgeman, 1991) the original picture alternates "with its modified version, at each time separated by brief blank fields.

Other global disruptions that have been used in investigations of change blindness are picture shifts (Blackmore, Brelstaff, Nelson, \& Troscianko, 1995), film cuts in motion pictures (Levin \& Simons, 1997) and physical occluders in real world situations (Simons \& Levin, 1998). Furthermore, change blindness can occur when the change takes place without an occluder, namely if a multitude of distracting local transients, known as "mudsplashes", are presented parallel to that produced by the change (O’Regan, Rensink, \& Clark, 1999) or when gradual changes occur slow enough not to give rise to the activation of the visual transient channels (Saiki, in press; Simons, Franconeri, \& Reimer, 2000).

Apart from the type of occlusion, various factors influence the phenomenon. Rensink et al. (1997) report that changes to objects of central interest (i.e. objects mostly mentioned in independent ratings) are detected more readily than changes to objects of marginal interest (mentioned by none). This means that the relevance of the object of change can play an important role in the detection. Also, the kind of change seems to be of importance: an object in a previously empty array is more likely to be detected than the replacement of one object by another. Stelmach, Bourassa, and Di Lollo (1984) found that adding an item to the visual array was more likely to be detected than deleting one. Change detection is further a function of knowledge and expertise of observers. In expert-novice comparison of change detection in football images, Werner and Thies (2000) showed that domain-specific expertise attenuates change blindness. These findings are supported by other data (e.g. Reingold, Charness, Pomplun, \& Stampe, 2001) that demonstrate experts' advantages in detection of changes to meaningful configurations. 


\subsection{Goals of the study}

In view of the variety of situations inducing change blindness (saccades, blinks, blanks, mudsplashes, gradual changes), the question arises how seriously this effect interferes with driving activity. Let us consider the mere number of blinks and saccades made during a day. Someone who blinks 12-15 times per minute (lasting $150 \mathrm{~ms}$ or more-see Barbato et al., 2000) while making approximately 4 saccades per second with an average duration of $35 \mathrm{~ms}$, experiences a global occlusion up to $18 \%$ of the time. Assuming that more than $50 \%$ of all collisions in road traffic can be lead back to a missing or delayed hazard perception (e.g. Nagayama, 1978), an explanation for at least some of these accidents could be change blindness: if a traffic light changes to red, just as the driver glances away or blinks - he/she may not notice this change, run over the red light and collide with a car or a pedestrian.

We were interested in finding out to what extent different occlusions may contribute to the failure of change detection in road traffic. Though hardly conceivable as a field investigation, the question can be addressed by laboratory experiments. Two experiments below describe change blindness effects in static and in dynamic (driving simulator) settings.

\section{Experiment 1: Change detection in static road traffic scenario}

\subsection{Method}

Twenty-four subjects (13 female and 11 male, aged 20-30 years) participated in the study. All subjects reported normal or corrected to normal vision. None of the subjects had participated in a change blindness study before. Each subject viewed 300 colour video stills of natural traffic situations presented on a 17 in. colour monitor with a viewing distance of approximately $60 \mathrm{~cm}$. In order to perform eye movement contingent changes to the scenes, the SR-Research Eyelink $^{\mathrm{TM}}$ recorder with a spatial accuracy of better than $1^{\circ}$ and $250 \mathrm{~Hz}$ sampling rate was used.

Using the forced choice paradigm, a display change could be produced randomly during three different occlusion modes_-blinks, saccades and artificial blanks (112 ms) — or during a fixation (as the control condition). To prevent blanks from serving as a cue, the same number of additional blanks without changes (catch trials) were produced. The changes could be either relevant or irrelevant with respect to the traffic safety (Fig. 1). Classification of relevance was realised by five independent judges with an inter-rater agreement better than 95\%. In addition, all kinds of changes were counterbalanced (inclusive deletions vs. insertions).

Subjects were asked to watch the scenes while trying to imagine being the driver of a car approaching the scene and to respond with a button press as soon as they noticed a change. Before the eye tracking equipment was fitted to the subject, a series of 20 test trials was presented to familiarize subjects with the procedure. After fitting and calibration of the eye tracker, subjects proceeded with the experiment. An automatic drift correction was performed every 5th trial, a recalibration took place every 30th trial. Subjects had a 5-min break after 100 and 200 trials. The order of presentation was partially counterbalanced across subjects. The whole experiment lasted approximately $75 \mathrm{~min}$, including preparation phase and two pauses. 

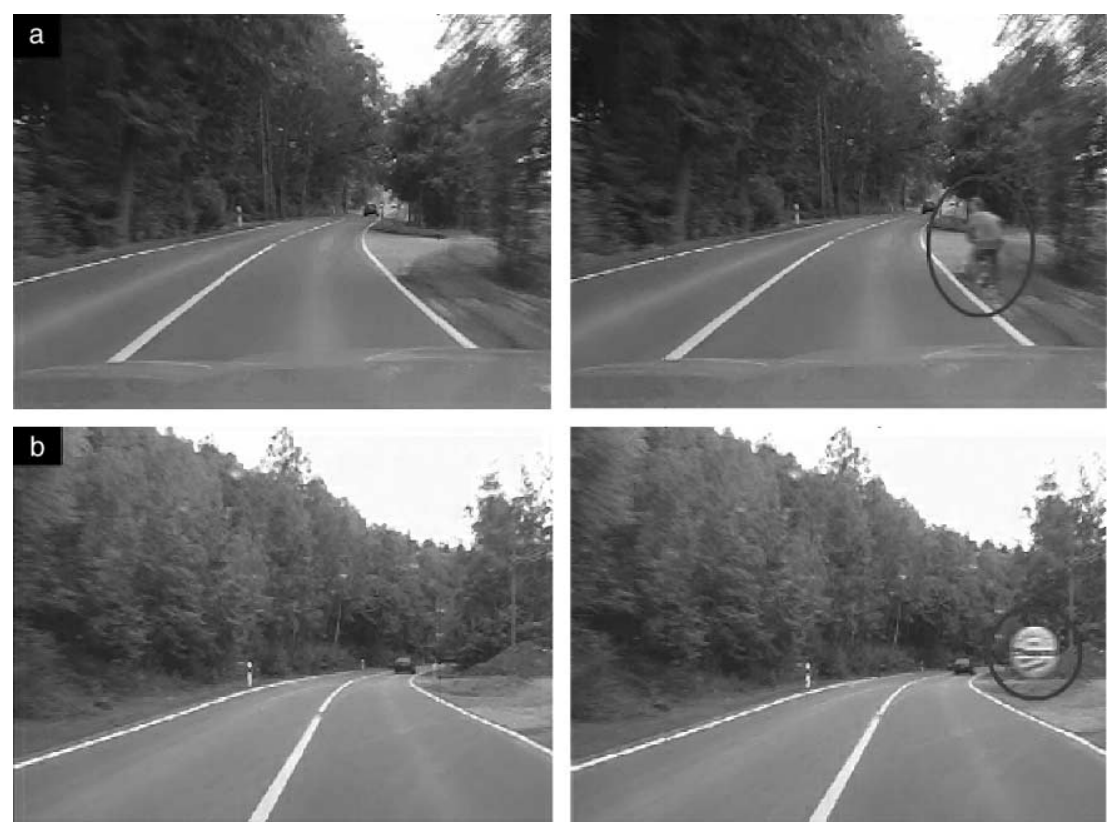

Fig. 1. Sample image-pairs used in the experiment: (a) relevant change: a bicyclist on the right side, (b) irrelevant change: an advertisement object on the right side.

\subsection{Results}

Eye movement data were inspected offline to decide whether they were recorded correctly or should be discarded. Observations were discarded when at the time of a change to image the intended occlusion (saccade, fixation or blink) was over. Five subjects with less than 10 observations in any of the six cells of the experimental design (occlusion type by relevance) were excluded from further data analysis, resulting in a final set of 19 subjects with a total of 4461 valid change detection observations. Guessing seemed not to play a role in the blank condition: the average false alarm rate in the 'no change' condition was 5.3\%, the highest individual rate among all the subjects was $8.3 \%$.

The detection rate of occluded relevant changes was much higher than that of irrelevant ones: $80 \%$ vs. $34 \%$, respectively $\left(F_{1,18}=360.32 ; p<.001\right)$. Relevance had also an effect on reaction time: on average, the relevant changes were detected $150 \mathrm{~ms}$ faster than the irrelevant ones $\left(F_{1,18}=6.32\right.$; $p<.013$ ). The detection rates and detection times for relevant and irrelevant changes are shown in Fig. 2; for each occlusion condition, bar colors indicating the two levels of relevance. We found the relevance effect in all occlusion conditions - for detection rate (for all comparisons, $p<.002$, Fig. 2(a)) as well as for detection time (for all comparisons, $p<.02$, Fig. 2(b)).

A closer look at the detection rate of only the relevant changes revealed a deterioration in detection for all occlusion conditions compared to fixation condition (for all comparisons, $p<.001$ ). No differences between the types of occlusion were found (for all comparisons, $p>.5$ ). With respect to the reaction time, we found similar differences: a slowdown of detection for all occlusions compared to the fixation condition (for all comparisons, $p<.001$ ) and no differences 


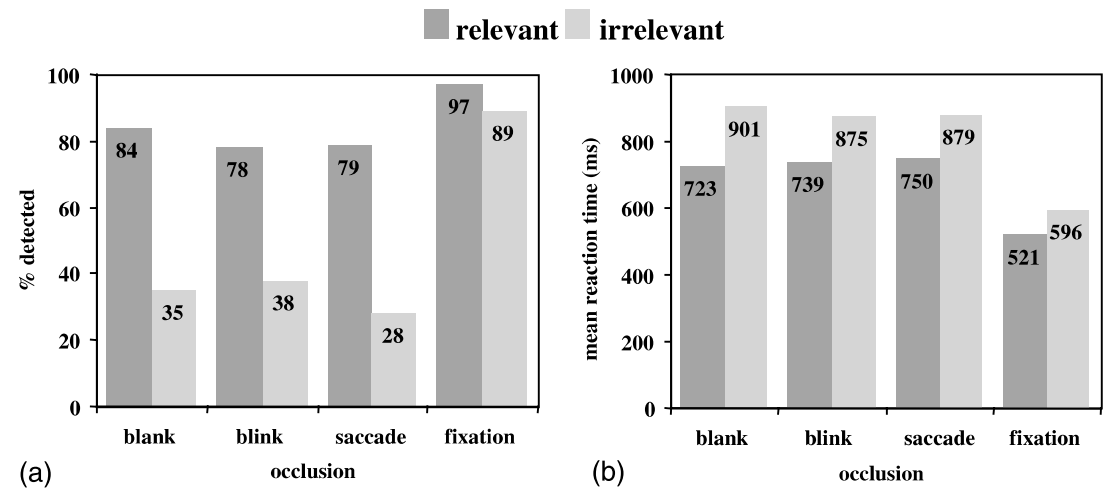

Fig. 2. (a) Detection rate by types of occlusion and relevance, (b) reaction time by types of occlusion and relevance.

between the occlusions (for all comparisons, $p>.8$ ): changes during occlusions reaction times were in average about $225 \mathrm{~ms}$ longer than in the non occlusion condition.

The results for detection rate and detection time of irrelevant changes are quite similar: both detection rate (for all comparisons, $p<.001$ ) and detection time (for all comparisons, $p<.001$ ) show differences in all conditions compared to the fixation condition. Reaction times for occluded changes were approximately $300 \mathrm{~ms}$ longer than for changes presented during fixations. No differences between the occlusion modes were found (for all comparisons, $p>.10$ ).

The data also revealed strong differences in dependence of the change types, namely differences between insertion and deletion. The detection probabilities and detection times for relevant insertions and deletions are shown in Fig. 3 separately for each mode of occlusion, bar colours indicating two levels of relevance.

In all occlusion modes, insertions were detected better than deletions, with the detection rate for insertions equal or above 90\% (for all comparisons, $p<.001$, Fig. 3(a)). Concerning the three types of occlusions however, again there was no difference in detection rate, neither for deletions nor for insertions (for all comparisons, $p>9$ ). In the same vein, insertions were detected in all
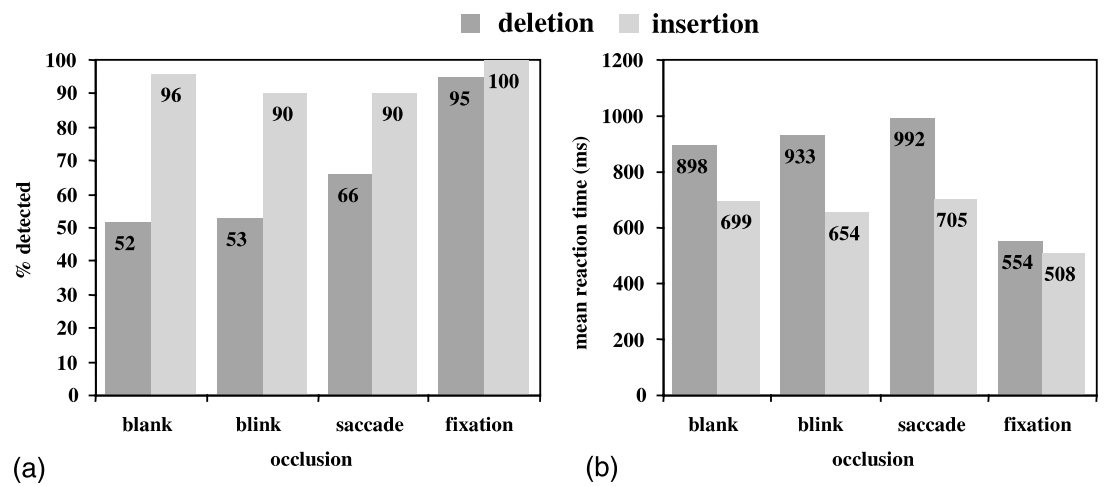

Fig. 3. Relevant changes only: (a) detection rate by types of occlusion and change kind, (b) reaction time by types of occlusion and change kind. 
occlusion modes faster than deletions (for all comparisons, $p<.001$, Fig. 3(b)), whereby a significant difference was found this time also in the fixation condition $(p<.04)$. Again, we did not find any difference between the occlusions (for all comparisons, $p>.2$ ). Compared to the fixation condition deletion slowdown in average was around $390 \mathrm{~ms}$ and insertion around $180 \mathrm{~ms}$. The effects of change type for irrelevant changes are analogous to that for the relevant ones-of course, due to the relevance effect the detection rate and reaction time differed.

\subsection{Discussion}

Our results show that all modes of occlusion have an essentially equal effect on change detection, also with respect to their interaction with other variables. Therefore, blanks can simulate blinks and saccades in static environments, at least if their duration is about $110 \mathrm{~s}$. The detection of occluded driving-relevant changes was much better than that of irrelevant ones. This is a correction to the bulk of change blindness demonstrations and good news with respect to traffic safety. Nevertheless, every fifth relevant change was missed in the static setting. As stated above, natural occlusions - saccades and blinks - occur at a relatively high rate. If to take into account this occlusion time with the miss rate of one fifth, then the driver might miss 1 of 25 relevant events in road traffic. Though relevant changes were detected faster than irrelevant ones it still took $225 \mathrm{~ms}$ longer to detect an occluded relevant change compared to a change without occlusion. At the speed of $50 \mathrm{~km}$ per hour, this means driving of about $4 \mathrm{~m}$ more before one would react to the change.

Concerning the kind of change, relevant insertions were detected more frequently and faster than relevant deletions. In a further study (Dornhoefer, Unema, \& Velichkovsky, in press), we were able to show that the detection is equally well in a range of eccentricity up to $10^{\circ}$ from the actual fixation point. It has to be mentioned also that in road traffic the more hazardous events are those that are related to the emergence of objects (even though objects temporally vanished from the field of view also can be highly relevant). From this point of view, the more hazardous changes are detected better and faster than the less hazardous ones. Despite the nearly perfect detection rate, relevant insertions were reported with a delay of approximately $180 \mathrm{~ms}$ in comparison to the fixation condition, which may be still a worrying difference.

Due to the fact that all these results were obtained with static scenes, their ecological validity and corresponding numerical calculations remains questionable however. To provide further evidence about the extent of change detection 'weakness' in road traffic we conducted a follow up driving simulation experiment, which is described below.

\section{Experiment 2: Change detection in a dynamic road traffic scenario}

\subsection{Method}

Twelve subjects (six female and six male, aged 19-31 years) participated in the study, for course credit or money. All subjects reported normal or corrected to normal vision and none of them had participated before in a change blindness study. 
The experiment was conducted using the 'SIRCA' driving simulator (developed and adapted for eye tracking experiments in cooperation with the ARTEC group at Institute of RoboticsUniversity of Valencia, Spain). With a resolution of 1280 by 1024 pixels, the simulator image was projected (via JVC DLA G11-video beamer) on a $2 \times 1.5 \mathrm{~m}$ silver screen. The frame rate of the simulator was about $20 \mathrm{~Hz}$. Subjects were seated at a distance of $3 \mathrm{~m}$ resulting in a view of approximately $32^{\circ}$ vertically and $24^{\circ}$ horizontally. The SR-Research Eyelink ${ }^{\mathrm{TM}}$ system was used to monitor subjects' eye movements. As the overall delay in detecting saccades by the system and the changes of the virtual environment was about $70 \mathrm{~ms}$, it was impossible in the experiment to produce gaze-depended changes during the natural occlusions.

A total of 50 different objects scanned from real life photographs (persons, animals, traffic signs, etc.) served as stimuli for the change detection. All changes (again the forced choice paradigm was used) were traffic-relevant insertions of objects, i.e. they belonged to the best detectable category of experiment 1 . For the purpose of realism, $65 \%$ of changes appeared on the right side from driver's point of view, the rest on the left side (within $8^{\circ}$ of the center of the screen). Furthermore, every change appeared at a distance that corresponded to $2 \mathrm{~s}$ of driving with the actual speed. Changes were presented randomly every 16-24 s either after a blanked screen or within a fixation, in proportion 40:60, respectively. In addition to blanks with changes, the same number of blanks without change (catch trials) was produced at random to avoid guessing. Fig. 4 shows two consecutive screenshots with an object inserted.

After a test drive of approximately $5 \mathrm{~min}$, subjects completed four consecutive 12 min-trials in the driving simulator. Pauses of $\approx 2$ min were used for a recalibration of the eye tracking system. Subjects were instructed to drive through an urban environment following traffic rules and keeping a velocity of approximately $50 \mathrm{~km}$ per hour. They were further asked to press a button on the steering wheel every time an object suddenly appeared on the road way. After the detection inserted object disappeared from the screen, otherwise it took exactly $2 \mathrm{~s}$ to pass the object.

\subsection{Results}

The average fixation duration in the experiment was $724 \mathrm{~ms}$ (with the mode of $626 \mathrm{~ms}$ ). An offline data analysis further yielded an average blank duration of $148 \mathrm{~ms}(\mathrm{SD}=13.59 \mathrm{~ms})$; variations
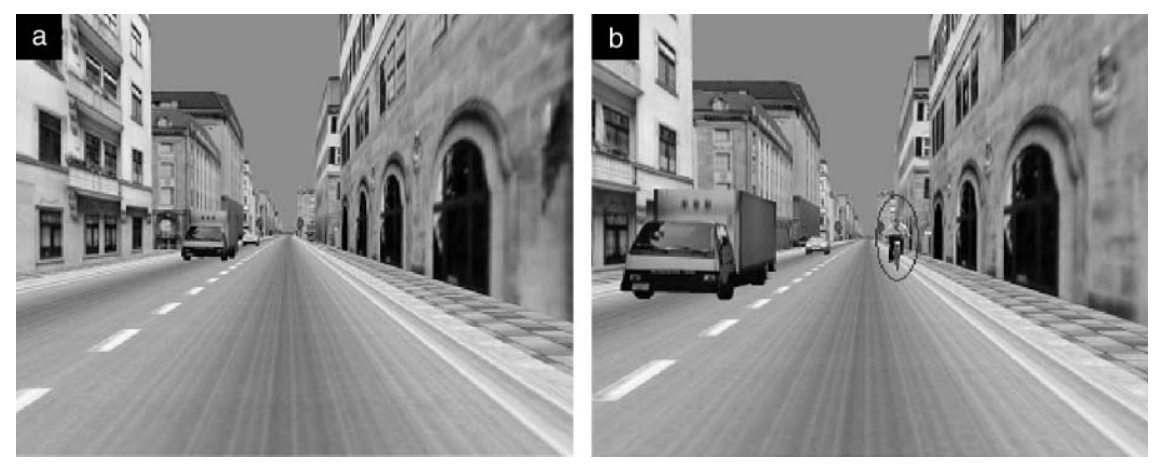

Fig. 4. An example of screenshots of the experiment 2: (a) before the blank, (b) after the blank: an oncoming bicyclist appears on the right lane. 
due to frame rate oscillations. The blink duration was approximately $170 \mathrm{~ms}$ in average. Changes during natural occlusions, i.e. blinks or saccades, were so rare that we separated them from the initial consideration. The final data set consisted of 1176 observations where objects were presented either within a fixation or within a blank.

In comparison of these two conditions, we suddenly found that the classical change blindness effect can be reversed. As can be seen in Fig. 5, there was a significant advantage-both in detection rate $\left(F_{1,11}=21.896 ; p<.002\right)$ as well as in detection time $\left(F_{1,11}=6.253 ; p<.030\right)$ for the blank condition compared to the fixation condition. There was no evidence of any excessive guessing during the blanks: the average false alarm rate in the 'no change' blank condition was $1.9 \%$; the highest rate among all the subjects was $4.4 \%$ only.

Of particular interest are the initially excluded observations of accidental changes produced during natural occlusions, i.e. blinks and saccades. After an eye movement analysis, 37 observations of changes strictly within saccades and 16 within blinks were selected for the further consideration from the perspective of speed-accuracy trade-off. While the change detection rate seemed to be lower in saccades than in blinks ( $87 \% \mathrm{vs} .100 \%$, respectively), subjects also needed approximately $80 \mathrm{~ms}$ more to react to changes if they were produced during saccades. These differences were significant $\left(t_{7}=3.772 ; p<.008\right)$. Change detection rates and reaction times for all conditions of this experiment are presented in Fig. 6.
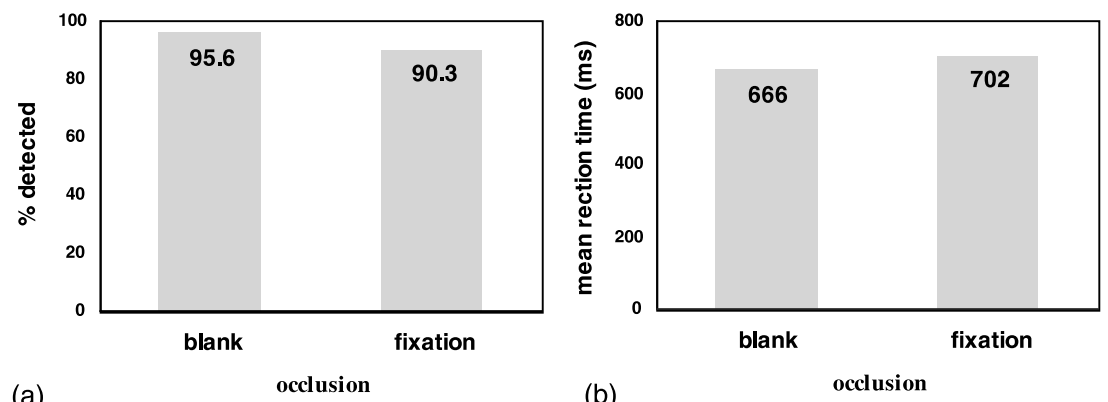

Fig. 5. (a) Detection rate for the blank and fixation condition, (b) mean reaction time for blank and fixation condition.

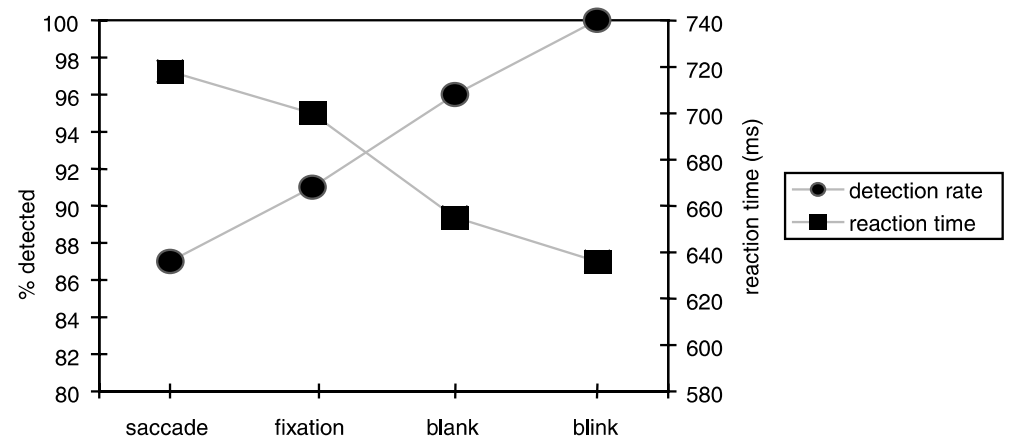

Fig. 6. Detection rate and reaction time for the three occlusion modes and the control, fixation condition. 
The major data of experiment 2, which are presented in a common format in the last figure, demonstrate a coherent variation in detection rate and reaction time across conditions. If considered in terms of speed-accuracy trade-off, these results can be summarised by the following conclusion: as the modes of occlusion, blinks and blanks are related to the best performance in change detection, saccades - to the worst, with the detection in the control fixation condition lying somewhere in-between.

\subsection{Discussion}

As the first investigation of change detection in a dynamic setting with a control of different occlusion modes, experiment 2 brought about several expected and less expected results. It is reasonable to consider them with reference to data from our first experiment conducted in a static setting. Though change detection rates of the present experiment appear to be generally high, it is to remind that they are detection of road-traffic relevant insertions. In this category of changes, fixation (base-line) data of experiment 1 (see Fig. 3) demonstrate an even better detection of 100\% accuracy and $508 \mathrm{~ms}$ reaction time. The difference in performance between the two experiments under the control condition can be attributed to only one factor, namely dynamics of situation.

The main result of experiment 2 is of course the largely different effects of the occlusion modes. Let us take, for instance, change detection in blanks, commonly used to produce change blindness. In the dynamic setting, the blank occlusion leads to an improvement in accuracy and speed of detection. What is the reason for this astonishing reversal of 'change blindness'? One could suppose that in the dynamic setting blanks serve as a cue telling the subject if not where then at least when to search for change. There is compelling evidence against such an explanation however. Firstly, false alarm rate was low in blanks. Secondly and more important, the cuing hypothesis cannot explain the perfect change detection with blinks. In experiment 2, changes were not contingent on blinks and in addition during blinks they were extremely rare. This is why we prefer another approach by stressing one similarity of otherwise so different blanks and blinks in one parameter - the duration of occlusion.

This parameter also differentiates blinks and blanks as a whole from saccades. With their duration of about $40 \mathrm{~ms}$, saccadic occlusions lead to a relatively low rate of change detection as well as slow reaction times. The question is then, what may happen on the continuum of occlusion durations from 40 to $150 \mathrm{~ms}$ that interact with dynamics of situation and overturn 'change blindness' effect? A preliminary answer can be found in research on visual motion and causality perception (Michotte, 1946/1963) that demonstrates that occlusions of about $100 \mathrm{~ms}$ and more disrupt the perceptual continuity of visual events, transforming them to a short static snapshot.

In a static environment, global occlusions obscure the local transient caused by a change and thereby prevent attention from being attracted to it. A dynamic situation is changing constantly. In contrast to static scenes, the multitude of distracting transients makes it likely that attention is equally driven to all potential regions of change. Hence, any interruption that is sufficiently long (but certainly not too long, as with fatigue blinks become as long as $400 \mathrm{~ms}$ - Stern, Boyer, \& Schroeder, 1994) can counteract the attentional load imposed by the flow of moving gradients and contours. This enables subjects to see that a change has taken place. In other words, we consider our dynamic data in line with the puzzling masking-of-masking effects (e.g. Dember \& Stefl, 1972) that are related to a recovery of the visual target when masked by several masking stimuli instead 
of one. There is also at least one recent observation on a combining of two occlusions in change blindness studies (Deubel \& Schneider, in press): changes within saccades followed by blanks were actually easier to detect than in any of these conditions alone.

\section{Conclusions}

Changes to environment are more difficult to detect in dynamic road-traffic scenarios in comparison to static situations. The differences are not only of a quantitative but also of a qualitative kind. First of all, only a narrow class of changes to a scene such as relevant insertions is of practical importance for driving. Within this class of changes, we systematically found the highest accuracy and fastest reaction times. Secondly, different modes of occlusion, among them such omnipresent natural occlusions as saccadic eye movements and blinks, selectively interfere with perception and attention in motion flow fields and, as it seems, may even improve detection.

There are only few empirical studies on change detection errors in real world (Simons \& Levin, 1998), simulated environments (Wallis \& Bülthoff, 2000; Shinoda, Hayhoe, \& Shrivastava, 2001) and motion pictures (Levin \& Simons, 1997). Their extension to driving scenarios will provide a better understanding of event perception in dynamically changing displays. Current studies in dynamic settings however largely differ with respect to their methods (see also Saiki, in press). Although the nature of the enhancement effect discovered in our experiments clearly needs further investigation, we meanwhile recommend great caution in extrapolating results from "change blindness" studies with blanks, static images and irrelevant changes to the more realistic driving situations.

\section{Acknowledgements}

Major part of the work described in this chapter was supported by research grants of the BMW AG, Munich. We would like to thank Pieter J.A. Unema for his participation in the first experiment and Marcos Fernandez Marin for the help in connecting the SIRCA-simulator to the SR-Research Eyelink ${ }^{\mathrm{TM}}$ system. We also thank two anonymous reviewers for helpful comments on an earlier draft.

\section{References}

Barbato, G., Ficca, G., Muscettola, G., Fichele, M., Beatrice, M., \& Rinaldi, F. (2000). Diurnal variation in spontaneous eye-blink rate. Psychiatry Research, 93(2), 145-151.

Blackmore, S. J., Brelstaff, G., Nelson, K., \& Troscianko, T. (1995). Is the richness of our visual world an illusion? Transsaccadic memory for complex scenes. Perception, 24, 1075-1081.

Dember, W. N., \& Stefl, M. (1972). Backward enhancement? Science, 175(4017), 93-95.

Deubel, H., \& Schneider, W. X. (in press). Selective information processing around the time of the saccade. In J. Hyönä, R. Radach, \& H. Deubel (Eds.), The mind's eye: cognitive and applied aspects of eye movements, progress in brain research. Oxford, UK: Elsevier.

Dornhoefer, S. M., Unema, P., \& Velichkovsky, B. M. (in press). Blanks, blinks and saccades: How blind we really are for relevant visual events. In J. Hyönä, D. Munoz, W. Heide, \& R. Radach (Eds.), The brain's eye: neurobiological and clinical aspects of oculomotor research, progress in brain research. Oxford, UK: Elsevier. 
Grimes, J. (1996). On the failure to detect changes in scenes across saccades. In K. Akins (Ed.), Perception (Vancouver Studies in Cognitive Sciences) (vol. 2) (pp. 89-110). New York: Oxford University Press.

Levin, D. T., \& Simons, D. J. (1997). Failure to detect changes to attended objects in motion pictures. Psychonomic Bulletin and Review, 4(4), 501-506.

Macknik, S., Fisher, B., \& Bridgeman, B. (1991). Flicker distorts visual space constancy. Vision Research, 31, 20572064.

Michotte, A., (1946/1963). The perception of casuality (T.R. Miles, E. Miles, Transl.). London: Methuen.

Nagayama, Y. (1978). Role of visual perception in driving. IATSS Research, 2, 64-73.

O’Regan, J. K., Deubel, H., Clark, J. J., \& Rensink, R. A. (2000). Picture changes during blinks: looking without seeing and seeing without looking. Visual Cognition, 7(1-3), 191-211.

O’Regan, J. K., Rensink, R. A., \& Clark, J. J. (1999). Change-blindness as a result of mudsplashes. Nature, 398(6722), 34.

Reingold, E. M., Charness, N., Pomplun, M., \& Stampe, D. M. (2001). Visual span in expert chess players: Evidence from eye movements. Psychological Science, 12(1), 48-55.

Rensink, R. A., O'Regan, J. K., \& Clark, J. J. (1997). To see or not to see the need for attention to perceive changes in scenes. Psychological Science, 8, 368-373.

Saiki, J. (in press). Multiple-object permanence tracking: Limitation in maintenance and transformation of perceptual objects. In J. Hyönä, D. Munoz, W. Heide, \& R. Radach (Eds.), The brain's eyes: Neurobiological and clinical aspects of oculomotor research, progress in brain research. Oxford, UK: Elsevier.

Shinoda, H., Hayhoe, M. M., \& Shrivastava, A. (2001). What controls attention in natural environments? Vision Research, 41(25-26), 3535-3545.

Simons, D. J. (1996). In sight, out of mind: when object representations fail. Psychological Science, 7(5), 301-305.

Simons, D. J., Franconeri, S. L., \& Reimer, R. L. (2000). Change blindness in the absence of visual disruption. Perception, 29, 1143-1154.

Simons, D. J., \& Levin, D. T. (1997). Change blindness. Trends in Cognitive Sciences, 1(7), 261-267.

Simons, D. J., \& Levin, D. T. (1998). Failure to detect changes to people in a real-world interaction. Psychonomic Bulletin and Review, 5(4), 644-649.

Stelmach, L. B., Bourassa, C. M., \& Di Lollo, V. (1984). Detection of stimulus change: The hypothetical roles of visual transient responses. Perception and Psychophysics, 35(3), 245-255.

Stern, J., Boyer, D., \& Schroeder, D. (1994). Blink rate: a possible measure of fatigue. Human Factors, 36(2), 285-297.

Wallis, G., \& Bülthoff, H. H. (2000). What's in scene and not seen. Visual Cognition, 7(1-3), 175-190.

Werner, S., \& Thies, B. (2000). Is change blindness attenuated by domain-specific expertise? An expert-novices comparison of change detection in football images. Visual Cognition, 7(1-3), 163-173. 